\title{
Haar null sets and the consistent reflection of non-meagreness
}

\author{
Márton Elekes* and Juris Steprāns ${ }^{\dagger}$ \\ February 5, 2013
}

\begin{abstract}
A subset $X$ of a Polish group $G$ is called Haar null if there exists a Borel set $B \supset X$ and Borel probability measure $\mu$ on $G$ such that $\mu(g B h)=0$ for every $g, h \in G$. We prove that there exist a set $X \subset \mathbb{R}$ that is not Lebesgue null and a Borel probability measure $\mu$ such that $\mu(X+t)=0$ for every $t \in \mathbb{R}$. This answers a question from David Fremlin's problem list by showing that one cannot simplify the definition of a Haar null set by leaving out the Borel set $B$. (The answer was already known assuming the Continuum Hypothesis.)

This result motivates the following Baire category analogue. It is consistent with $Z F C$ that there exist an abelian Polish group $G$ and a Cantor set $C \subset G$ such that for every non-meagre set $X \subset G$ there exists a $t \in G$ such that $C \cap(X+t)$ is relatively non-meagre in $C$. This essentially generalises results of Bartoszyński and Burke-Miller.
\end{abstract}

\section{Introduction}

\section{$1.1 \quad$ Haar null sets}

Let us first give some motivation for studying Haar null sets in non-locally compact groups. The following definition is due to Christensen [4] (and later

\footnotetext{
*Partially supported by the Hungarian Scientific Foundation grants no. 72655, 61600, 83726 and János Bolyai Fellowship.

$\dagger$ Partially supported by NSERC of Canada.

MSC codes: Primary 28C10, 03E35 Secondary 03E17, 22C05, 28A78

Key Words: Haar null, Christensen, non-locally compact Polish group, packing dimension, Problem FC on Fremlin's list, forcing, generic real
} 
independently to Hunt, Sauer and Yorke [12]).

Definition 1.1 A subset $X$ of a Polish group $G$ is called Haar null if there exists a Borel set $B \supset X$ and Borel probability measure $\mu$ on $G$ such that $\mu(g B h)=0$ for every $g, h \in G$.

(All measures in the paper are assumed to be countably additive.)

The above definition is justified by the following theorem.

Theorem 1.2 (Christensen [4]) A subset of a locally compact Polish group is Haar null in the above sense iff it is of Haar measure zero.

There is a huge literature devoted to Haar null sets, e.g. they form a $\sigma$-ideal, but Fubini's theorem fails, etc. See for example the work of Mycielski, Dougherty, Solecki, Matoušková, Zajičcek, Duda, Dodos, Shi, Banakh, Holický, etc. (Note that some authors use the term shy for Haar null, and prevalent for co-Haar null.)

Haar null sets turned out to be very useful in numerous areas of mathematics.

First, they can serve as a measure counterpart of some Baire category arguments, even when no actual measure is present. Often the typical behaviour with respect to this notion of Haar null dramatically differs from the one with respect to Baire category. For instance,

Theorem 1.3 (Hunt [11]) $\left\{f \in C[0,1]: \exists x \exists f^{\prime}(x) \in \mathbb{R}\right\}$ is meagre and Haar null.

But:

Theorem 1.4 (Zajíček [18]) $\left\{f \in C[0,1]: \exists x \exists f^{\prime}(x) \in[-\infty, \infty]\right\}$ is meagre but not Haar null.

The next two theorems concerning the cycle structure of permutations of the integers also illustrate the striking difference between meagre and Haar null.

Theorem 1.5 (Folklore) Comeagre many elements of $S_{\infty}$ have infinitely many cycles of length $n$ for every $n \in \omega$ and no infinite cycles.

But: 
Theorem 1.6 (Dougherty-Mycielski [8]) Haar-a.e. element of $S_{\infty}$ has infinitely many infinite cycles and finitely many finite cycles.

Secondly, Haar null sets show up naturally as exceptional small sets. For example,

Theorem 1.7 (Christensen [5]) Let $X$ be a separable Banach space and $f: X \rightarrow \mathbb{R}$ a Lipschitz function. Then $f$ is Gâteaux differentiable outside of a Haar null set.

\subsection{Problem FC on Fremlin's list}

After motivating this notion, let us now describe the starting point of the present paper, which is Problem FC on Fremlin's list1 1 . The question essentially asks: "But do we need this Borel set $B$ in the definition of Haar null?" The question makes sense for locally compact groups as well, so it was formulated originally for $\mathbb{R}$ for simplicity. From now on we will slightly abuse notation and identify the Borel measure $\mu$ with the outer measure generated by it.

Problem 1.8 Let $X \subset \mathbb{R}$, and let $\lambda$ denote Lebesgue (outer) measure.

$\lambda(X)=0 \Longleftrightarrow \exists$ a Borel probability measure $\mu$ s.t. $\forall t \in \mathbb{R} \mu(X+t)=0$ ?

Note that the left-to-right implication is obvious. Problem FC also asks if we can find a counterexample $X$ to the right-to-left implication when $\mu=$ $\mu_{\text {Cantor }}$ is the usual Cantor measure ("coin tossing measure") on the Cantor set.

Problem 1.9 Let $X \subset \mathbb{R}$.

$$
\forall t \in \mathbb{R} \mu_{\text {Cantor }}(X+t)=0 \Longrightarrow \lambda(X)=0 ?
$$

In fact, Fremlin remarks that the answer to both questions is negative if we assume the Continuum Hypothesis. Let us now prove this for the sake of completeness.

\footnotetext{
${ }^{1}$ Originally, Problem FC consisted of Problem 1.8 and Problem 1.9, but after we had solved Problem 1.8 and communicated our results to Prof. Fremlin, he has modified the problem by mentioning our theorem and erasing the corresponding half of the problem.
} 
Claim 1.10 Assume the Continuum Hypothesis. Then the answers to Problem 1.8 and Problem 1.9 are in the negative, that is, $\exists X \subset \mathbb{R}$ with $\lambda(X)>0$ such that $\mu_{\text {Cantor }}(X+t)=0$ for every $t \in \mathbb{R}$.

Proof. Let $C$ denote the Cantor set. It suffices to construct an $X \subset \mathbb{R}$ with $\lambda(X)>0$ such that $C \cap(X+t)$ is countable for every $t \in \mathbb{R}$.

Let us enumerate the reals as

$$
\left\{t_{\alpha}: \alpha<\mathfrak{c}\right\}
$$

and the Borel sets of Lebesgue measure zero as

$$
\left\{Z_{\alpha}: \alpha<\mathfrak{c}\right\}
$$

At stage $\alpha$ let us pick an

$$
x_{\alpha} \in \mathbb{R} \backslash\left(\cup_{\beta<\alpha}\left(C-t_{\beta}\right) \cup Z_{\alpha}\right) .
$$

Set

$$
X=\left\{x_{\alpha}: \alpha<\mathfrak{c}\right\} .
$$

Then $x_{\alpha} \notin Z_{\alpha}$ shows that $\lambda(X)>0$. Moreover, for $\alpha>\beta, x_{\alpha}+t_{\beta} \notin C$ implies $C \cap\left(X+t_{\beta}\right) \subset\left\{x_{\alpha}: \alpha \leq \beta\right\}$, hence $C \cap\left(X+t_{\beta}\right)$ is countable.

Remark 1.11 In fact we only used $\operatorname{cov}(\mathcal{N})=\operatorname{cof}(\mathcal{N})$ (see 2 for the definitions). A more involved argument using ideas similar to the ones in Section 2 shows that $\operatorname{non}(\mathcal{N})=\mathfrak{c}$ also suffices.

Therefore the real questions are whether we can find counterexamples in $\mathrm{ZFC}$, that is, without resorting to extra set-theoretic assumptions. Our first main goal will be to show in Section 2 (Corollary 2.4) that Problem 1.8 actually has a negative answer in ZFC.

Theorem 1.12 (First Main Theorem) Problem 1.8 has a negative answer, that is, there exist $X \subset \mathbb{R}$ with $\lambda(X)>0$ and a Borel probability measure $\mu$ such that $\mu(X+t)=0$ for every $t \in \mathbb{R}$.

Before formulating our second main result, which involves more set theory, let us introduce some terminology. 
Definition 1.13 Let $X \subset \mathbb{R}$ with $\lambda(X)>0$ and $\mu$ be a Borel probability measure. We say that $\mu$ reflects the positive measure of $X$ if there exists $t \in \mathbb{R}$ such that $\mu(X+t)>0$.

Hence, taking Claim 1.10 into account, we can reformulate Fremlin's second problem as

Problem 1.14 Is it consistent that $\mu_{\text {Cantor }}$ reflects the positive measure of every $X$ ?

This problem is still open, but our second main goal will be to give an affirmative answer to a category analogue in Section 3 .

Now we describe this category analogue in a bit more detail. Theorem 1.12 states that there exists a $\mu$ that does not reflect. Problem 1.14, which is still open, asks if a fixed measure $\left(\mu_{\text {Cantor }}\right)$ consistently reflects. So it is natural to ask the same question about other (fixed) measures.

Problem 1.15 Is it consistent that there exist an atomless singular Borel probability measure $\mu$ such that for every $X \subset \mathbb{R}$ with $\lambda(X)>0$ there exists $t \in \mathbb{R}$ such that $\mu(X+t)>0$ ?

Unfortunately, this is also open. In order to get a better understanding of the problem, let us consider the following category analogue. Recall that a set is a Cantor set if it is homeomorphic to the classical middle-thirds Cantor set.

Problem 1.16 Is it consistent that there exist a Cantor set $K$ such that for every non-meagre set $X \subset \mathbb{R}$ there exists a $t \in \mathbb{R}$ such that $K \cap(X+t)$ is relatively non-meagre in $K$ ?

There are numerous Polish groups that are called "the reals" in set theory. For example, certain results are simpler to prove in $\mathbb{Z}_{2}^{\omega}$ (the Cantor group) than in $\mathbb{R}$, but usually it is only a technical issue to convert the proofs to $\mathbb{R}$ (note that the dyadic expansion shows that $[0,1)$ with addition modulo 1 and $\mathbb{Z}_{2}^{\omega}$ are very similar, the only difference is the presence of carried digits). For technical reasons we will replace $\mathbb{R}$ with

$$
\mathbb{R}^{\prime}=\prod_{m \in \omega} \mathbb{Z}_{m+3}
$$


where $\mathbb{Z}_{m+3}=\{0,1, \ldots, m+2\}$ with addition modulo $m+3$. Again this group can also be considered as "the reals", since this is essentially an expansion with an "increasing base", as shown by the following map.

$$
\left(n_{m}\right)_{m \in \omega} \in \mathbb{R}^{\prime} \mapsto \sum_{m \in \omega} \frac{n_{m}}{(m+3) !} \in \mathbb{R},
$$

which is the analogue of the map $\left(n_{m}\right)_{m \in \omega} \in \mathbb{Z}_{2}^{\omega} \mapsto \sum_{m \in \omega} \frac{n_{m}}{2^{m}} \in \mathbb{R}$ that connects the dyadic form of the reals with the usual one.

The second main goal will be to give an affirmative answer to Problem 1.16 in Section 3 (Theorem 3.35) for this slightly modified underlying group.

Theorem 1.17 (Second Main Theorem) It is consistent that there exist a Cantor set $C_{E K} \subset \mathbb{R}^{\prime}$ such that for every non-meagre set $X \subset \mathbb{R}^{\prime}$ there exists a $t \in \mathbb{R}^{\prime}$ such that $C_{E K} \cap(X+t)$ is relatively non-meagre in $C_{E K}$.

Here

$$
C_{E K}=\prod_{m \in \omega}\left(\mathbb{Z}_{m+3} \backslash\{m+2\}\right) .
$$

This set was first considered by Erdős and Kakutani in [9], hence the notation.

The rest of the introduction is devoted to some closely related known results and historical remarks. If we forget about translates of a fixed Cantor set then the affirmative answer to Problem 1.16 is already known. Interestingly, the following theorem was proved independently in two papers.

Theorem 1.18 (Bartoszyński [1], Burke-Miller [3]) It is consistent that for every non-meagre set $X \subset \mathbb{R}$ there exists a Cantor set $K \subset \mathbb{R}$ such that $K \cap X$ is non-meagre in $K$.

The next theorem still does not use translates, but already finds Cantor sets of some special structure.

Theorem 1.19 (Ciesielski-Shelah [6]) For every non-meagre $X \subset 2^{\mathbb{N}} \times$ $2^{\mathbb{N}}$ there exists a homeomorphism $\varphi: 2^{\mathbb{N}} \rightarrow 2^{\mathbb{N}}$ such that $X \cap \operatorname{graph}(\varphi)$ is non-meagre in $\operatorname{graph}(\varphi)$.

Remark 1.20 The Burke-Miller paper also has a certain measure version of their result, and the Ciesielski-Shelah theorem also has some measure analogue (Rosłanowski-Shelah [17]), but these do not seem to say much about Problem 1.16. 


\section{The negative result: Solution to Problem 1.8}

The heart of the proof of this result is the following theorem, which is based on ideas from [7]. For the definition and basic properties of packing dimension, denoted by $\operatorname{dim}_{p} H$, see [10] or [15].

Theorem 2.1 Let $K^{\prime} \subset \mathbb{R}$ be a Cantor set with $\operatorname{dim}_{p} K^{\prime}<1$ and let $T \subset \mathbb{R}$ be such that $|T|<\mathfrak{c}$. Then $K^{\prime}+T$ contains no measurable set of positive measure.

Proof. Suppose on the contrary that $K^{\prime}+T$ contains a measurable set $P$ of positive measure. We may assume that $P$ is compact. By throwing away all portions (i.e. relatively open nonempty subsets) of measure zero, we may also assume that every portion of $P$ is of positive measure. In particular, $P$ has no isolated points. The idea of the proof will be to construct a Cantor set $P^{\prime} \subset P$ such that $P^{\prime} \cap\left(K^{\prime}+r\right)$ is finite for every $r \in \mathbb{R}$. This clearly suffices, since a Cantor set is of cardinality continuum and hence less than continuum many translates of $K^{\prime}$ cannot cover $P^{\prime}$, let alone $P$.

Let $N$ be a positive integer and let us define $F_{N}$ to be the set of $N$-tuples that can be covered by a translate of $K^{\prime}$, that is,

$$
F_{N}=\left\{\left(x_{0}, \ldots, x_{N-1}\right) \in \mathbb{R}^{N}: \exists t \in \mathbb{R} \text { such that }\left\{x_{0}, \ldots, x_{N-1}\right\} \subset K^{\prime}+t\right\} .
$$

An easy compactness argument shows that $F_{N}$ is closed. Reformulating the definition one can easily check that

$$
F_{N}=\left(K^{\prime}\right)^{N}+\mathbb{R}(1, \ldots, 1),
$$

where $(1, \ldots, 1)$ is a vector of $N$ coordinates, and the operations are Minkowski sum and Minkowski product. It is easy to see that $F_{N}$ is a Lipschitz image of $\left(K^{\prime}\right)^{N} \times \mathbb{R}$, and using that Lipschitz images do not increase packing dimension as well as $\operatorname{dim}_{p}(A \times B) \leq \operatorname{dim}_{p} A+\operatorname{dim}_{p} B$ and $\operatorname{dim}_{p} \mathbb{R}=1$ we obtain

$$
\operatorname{dim}_{p} F_{N} \leq N \operatorname{dim}_{p} K^{\prime}+1 .
$$

If we choose $N$ large enough, actually if $N>\frac{1}{1-\operatorname{dim}_{p} K^{\prime}}$, then $N \operatorname{dim}_{p} K^{\prime}+1<$ $N$, hence

$$
\operatorname{dim}_{p} F_{N}<N
$$

Let us fix such an $N$. 
Lemma 2.2 Let $J_{i} \subset \mathbb{R}(i<N)$ be closed intervals such that int $J_{i} \cap P \neq \emptyset$ $(i<N)$. Then there are disjoint closed intervals $I_{i} \subset J_{i}(i<N)$ such that int $I_{i} \cap P \neq \emptyset(i<N)$ and

$$
\prod_{i<N}\left(I_{i} \cap P\right) \cap F_{N}=\emptyset
$$

Proof. Since every portion of $P$ is of positive measure, we obtain $\lambda^{N}\left(\prod_{i<N}\left(\right.\right.$ int $\left.\left.J_{i} \cap P\right)\right)>0$, hence $\operatorname{dim}_{p}\left(\prod_{i<N}\left(\right.\right.$ int $\left.\left.J_{i} \cap P\right)\right)=N>\operatorname{dim}_{p} F_{N}$. Therefore $\left(\prod_{i<N}\left(\right.\right.$ int $\left.\left.J_{i} \cap P\right)\right) \backslash F_{N} \neq \emptyset$, and, since $F_{N}$ is closed, $\prod_{i<N}\left(\right.$ int $J_{i} \cap$ $P$ ) contains a non-empty relatively open set avoiding $F_{N}$. This open set contains a basic open set, so there are open intervals $J_{i}^{\prime} \subset$ int $J_{i}(i<N)$ intersecting $P$ such that $\prod_{i<N}\left(J_{i}^{\prime} \cap P\right) \cap F_{N}=\emptyset$.

Finally, since $P$ has no isolated points, it is easy to shrink every $J_{i}^{\prime}$ to a closed interval $I_{i}$ such that they become disjoint but their interiors still meet $P$. This finishes the proof of the lemma.

Now we return to the proof of the theorem. All that remains is to construct $P^{\prime}$. We will actually prove

$$
\left|P^{\prime} \cap\left(K^{\prime}+r\right)\right|<N \text { for every } r \in \mathbb{R} \text {. }
$$

We construct a usual Cantor scheme, where the $k^{\text {th }}$ level $\mathcal{L}_{k}$ will have the following properties for all $k \in \omega$.

(1) $\mathcal{L}_{k}$ consist of $N^{k}$ many disjoint closed intervals,

(2) $\forall I \in \mathcal{L}_{k+1} \exists I^{\prime} \in \mathcal{L}_{k}: I \subset I^{\prime}$,

(3) $\forall I \in \mathcal{L}_{k}$ there are $N$ many $I^{\prime} \in \mathcal{L}_{k+1}$ with $I^{\prime} \subset I$,

(4) $\forall I \in \mathcal{L}_{k}:$ int $I \cap P \neq \emptyset$,

(5) $\forall I \in \mathcal{L}_{k}: \operatorname{diam} I \leq \frac{1}{k+1}$

(6) If $I_{0}, \ldots, I_{N-1} \in \mathcal{L}_{k}$ are distinct then $\prod_{i<N}\left(I_{i} \cap P\right) \cap F_{N}=\emptyset$.

(Note that the intervals in (6) are not necessarily subsets of the same $I^{\prime} \in$ $\mathcal{L}_{k-1}$.) Assume first that such a Cantor scheme exists, and define

$$
P^{\prime}=\bigcap_{k \in \omega} \bigcup \mathcal{L}_{k}
$$


It is easy to see that $P^{\prime}$ is a Cantor set $([13])$, while the closedness of $P$, (44) and (5) imply $P^{\prime} \subset P$. Let $x_{0}, \ldots, x_{N-1}$ be $N$ distinct points in $P^{\prime}$. Clearly, there is a $k$ and distinct intervals $I_{0}, \ldots, I_{N-1} \in \mathcal{L}_{k}$ such that $x_{i} \in I_{i}(i<N)$. Then (6) shows that $\left\{x_{0}, \ldots, x_{N-1}\right\}$ cannot be covered by a translate of $K^{\prime}$, which proves (2.1).

Finally, let us prove by induction that such a Cantor scheme exists. Let $\mathcal{L}_{0}=\{I\}$, where $I$ is an arbitrary closed interval of length at most 1 whose interior meets $P$. Assume that $\mathcal{L}_{k}$ have already been constructed with the required properties. Let $\mathcal{L}_{k+1}^{\prime}$ be a family of disjoint closed intervals of length at most $\frac{1}{k+2}$ whose interiors meet $P$ such that each $I \in \mathcal{L}_{k}$ contains $N$ members of $\mathcal{L}_{k+1}^{\prime}$. Then recursively shrinking these intervals by applying Lemma $2.2\left(\begin{array}{c}N^{k+1} \\ N\end{array}\right)$ times to all the possible $N$-tuples of distinct intervals we obtain $\mathcal{L}_{k+1}$ satisfying all assumptions. This concludes the proof of the theorem.

Theorem 2.3 Let $K \subset \mathbb{R}$ be a Cantor set with $\operatorname{dim}_{p} K<1 / 2$. Then there exists $X \subset \mathbb{R}$ with $\lambda(X)>0$ such that $|K \cap(X+t)| \leq 1$ for every $t \in \mathbb{R}$.

Proof. As above, let us enumerate the Borel sets of Lebesgue measure zero as $\left\{Z_{\alpha}: \alpha<\mathfrak{c}\right\}$. Since $K-K$ is a Lipschitz image of $K \times K$, we obtain $\operatorname{dim}_{p}(K-K)<1$. At stage $\alpha$ let us pick an

$$
x_{\alpha} \in \mathbb{R} \backslash\left(\left((K-K)+\left\{x_{\beta}: \beta<\alpha\right\}\right) \cup Z_{\alpha}\right) .
$$

This is indeed possible by the above theorem applied to $K^{\prime}=K-K$. Set

$$
X=\left\{x_{\alpha}: \alpha<\mathfrak{c}\right\}
$$

Then $x_{\alpha} \notin Z_{\alpha}$ shows that $\lambda(X)>0$. We still have to check that $\mid K \cap(X+$ $t) \mid \leq 1$ for every $t \in \mathbb{R}$. Let $x_{\alpha}, x_{\beta} \in X$ with $\alpha>\beta$, and let us assume $x_{\alpha}+t, x_{\beta}+t \in K$. Then $t \in K-x_{\beta}, x_{\alpha} \in K-\left(K-x_{\beta}\right)=(K-K)+x_{\beta}$, contradicting the choice of $x_{\alpha}$.

From this we easily obtain our first main theorem (Theorem 1.12) as a corollary.

Corollary 2.4 (First Main Theorem) Problem 1.8 has a negative answer, that is, there exist $X \subset \mathbb{R}$ with $\lambda(X)>0$ and a Borel probability measure $\mu$ such that $\mu(X+t)=0$ for every $t \in \mathbb{R}$. 
Proof. Indeed, let $K$ be any Cantor set with $\operatorname{dim}_{p} K<1 / 2$ (e.g. the "middle- $\alpha$ Cantor set" is such a set for $\alpha>1 / 2$ ). Let $\mu$ be any atomless Borel probability measure on $K$. Then by the above theorem $\mu$ does not reflect the positive measure of $X$.

\section{The positive result}

\subsection{The forcing poset}

The skeleton of the proof of the second main result will be borrowed from Bartoszyński's paper [1].

Notation 3.1 Set $\Sigma=\bigcup_{l \in \omega} \prod_{m<l} \mathbb{Z}_{m+3}$, that is, for $s \in \Sigma$ the sets $[s]=$ $\left\{x \in \prod_{m \in \omega} \mathbb{Z}_{m+3}: s \subset x\right\}$ form the usual clopen base of $\prod_{m \in \omega} \mathbb{Z}_{m+3}$. The symbol $|s|$ will denote the length of the sequence $s \in \Sigma$, that is, the cardinality of $\operatorname{dom}(s)$.

Recall that $\forall^{\infty}$ means 'for all but finitely many', and $[\omega]^{<\omega}$ denotes the set of finite subsets of $\omega$.

Definition 3.2 Let $s \in \Sigma$ and $k \in \omega$. Then $S: \omega \backslash \operatorname{dom}(s) \rightarrow[\omega]^{<\omega}$ is $a$ finite $k$-slalom above $s$, if

(1) $\forall i \in \omega \backslash \operatorname{dom}(s) \quad|S(i)| \leq k$,

(2) $\forall^{\infty} i \in \omega \backslash \operatorname{dom}(s) S(i)=\emptyset$.

Definition 3.3 ht $(S)=\min \{i \in \omega \backslash \operatorname{dom}(s): \forall j \geq i S(j)=\emptyset\}$.

Definition 3.4 Let $S$ be a finite $k$-slalom above $s$ and $t \in \Sigma$. Then $t$ escapes $S$ if $t \supset s,|t| \geq \operatorname{ht}(S)$ and $\forall i \in[\operatorname{dom}(s), \operatorname{dom}(t)) t(i) \notin S(i)$.

Definition 3.5 Let $s \in \Sigma$ and $F \subset\{t \in \Sigma: t \supset s\}$. Then $F$ is $k$-fat above $s$, if for every finite $k$-slalom $S$ above $s$ there exists $t \in F$ escaping $S$.

Remark 3.6 It is easy to see that if $k \geq 1$ and $F$ is $k$-fat above $s$ then for every finite $k$-slalom $S$ above $s$ there exist arbitrarily long t's in $F$ escaping $S$. (Otherwise, just extend $S$ so that $\operatorname{ht}(S)$ is bigger than the length of all $t$ 's escaping $S$.) 
This immediately yields the following.

Fact 3.7 If $F$ is $k$-fat above $s$ and $V$ is a finite set then $F \backslash V$ is also $k$-fat above s.

Recall that $(\Sigma, \subset)$ is tree, that is, for the purposes of the present paper, a partially ordered set such that for each $\sigma \in \Sigma$ the set $\left\{\sigma^{\prime} \in \Sigma: \sigma^{\prime} \subset \sigma\right\}$ is finite. The $n^{\text {th }}$ level of a tree is the set of those points that have exactly $n$ smaller elements. If $\emptyset \neq T \subset \Sigma$ then $(T, \subset)$ is a tree itself.

Notation 3.8 If $t \in T$ then $\operatorname{succ}_{T}(t)$ will denote the set of immediate successors of $t$ in $T$. We simply write $\operatorname{succ}(t)$ when there is no danger of confusion. We say that $T$ has a unique root if it has a unique $\subset$-minimal element. In such cases this root will be denoted by $\operatorname{root}(T)$. For $t \in T$ let $T[t]=\{s \in T: s \supset t\}$.

Now we define our notion of forcing.

Definition 3.9 Let $p \in \mathbb{P}$ iff

(1) $p \subset \Sigma$,

(2) $p$ has a unique root (in particular, $p \neq \emptyset$ ),

(3) $\forall t \in p \operatorname{succ}_{p}(t)$ is 1 -fat above $t$.

(4) $\forall k \in \omega \forall^{\infty} t \in p \operatorname{succ}_{p}(t)$ is $k$-fat above $t$.

If $p, p^{\prime} \in \mathbb{P}$ then define

$$
p \leq_{\mathbb{P}} p^{\prime} \Longleftrightarrow p \subset p^{\prime}
$$

We will usually simply write $\leq$ for $\leq_{\mathbb{P}}$.

We will often use the following easy consequence of (41).

Fact 3.10 If $t \in p \in \mathbb{P}$ and $k \in \omega$ then there exists $s \in p$ such that $\operatorname{succ}_{p}(s)$ is $k$-fat above $s$.

First we prove that $\mathbb{P}$ is nontrivial. 
Lemma 3.11 Let $k \geq 1$. If $H$ is $k$-fat above $t$ then $H$ contains a subset consisting of pairwise incompatible sequences that is $(k-1)$-fat above $t$.

Proof. Let $\left\{S_{n}\right\}_{n \in \omega}$ be an enumeration of the $(k-1)$-slaloms above $t$. It clearly suffices to recursively pick pairwise incomparable $t_{n}$ 's in $H$ such that $\left|t_{n}\right|>0$ is strictly increasing and $t_{n}$ escapes $S_{n}$. Suppose $\left\{t_{m}\right\}_{m<n}$ has already been constructed in such a manner. Then we can form a $k$-slalom by adding the "last elements of the $t_{m}$ 's" to $S_{n}$, that is, let

$$
S_{n}^{\prime}(i)= \begin{cases}S_{n}(i) \cup\left\{t_{m}\left(\left|t_{m}\right|-1\right)\right\} & \text { if } i=\left|t_{m}\right|-1, \\ S_{n}(i) & \text { otherwise. }\end{cases}
$$

Then $S_{n}^{\prime}$ is indeed a $k$-slalom, hence we can choose a $t_{n}$ escaping it. By Remark [3.6 we may assume $\left|t_{n}\right|>\left|t_{n-1}\right|$. Then the definition of $S_{n}^{\prime}$ shows that $t_{n}$ is incomparable to $t_{m}$ for every $m<n$, and we are done.

Lemma $3.12 \mathbb{P} \neq \emptyset$.

Proof. We inductively construct the levels $l_{n}$ of a tree $p \subset \Sigma$ such that

(1) $\left|l_{0}\right|=1$,

(2) every $l_{n}$ consists of pairwise incomparable sequences,

(3) $\forall n \forall s \in l_{n+1} \exists t \in l_{n} t \varsubsetneqq s$,

(4) $\forall n \forall t \in l_{n} l_{n+1} \cap \Sigma[t]$ is $|t|+1$-fat above $t$.

By (3) we clearly have $|t| \geq n$ for every $t \in l_{n}$. Moreover, since $\Sigma$ is a finitely branching tree, (2) implies that $\forall k \forall^{\infty} t \in l_{n}|t| \geq k$. Using these two facts and (4) it is easy to see that if such a sequence $\left\{l_{n}\right\}_{n}$ exists then $p=\bigcup_{n} l_{n} \in \mathbb{P}$.

Let us now check that we can carry out this induction. Suppose that such an $\left\{l_{m}\right\}_{m \leq n}$ has already been constructed. It is easy to see that $\Sigma[t]$ is $|t|+2$-fat above $t$ for every $t \in \Sigma$. Hence, using Lemma 3.11, for every $t \in l_{n}$ we can pick $H_{t} \subset \Sigma[t]$ consisting of pairwise incomparable elements that is $|t|+1$-fat above $t$. (We may assume $t \notin H_{t}$.) Then setting $l_{n+1}=\bigcup_{t \in l_{n}} H_{t}$ completes the proof.

The following fact is immediate. 
Fact 3.13 If $t \in p \in \mathbb{P}$ then $p[t] \in \mathbb{P}, p[t] \leq p$, and $\operatorname{root}(p[t])=t$.

This easily yields the following three statements.

Corollary 3.14 $\mathbb{P}$ is a separative partial order, and there are incompatible conditions below every condition.

Corollary 3.15 $\left\{p \in \mathbb{P}: \operatorname{succ}_{p}(\operatorname{root}(p))\right.$ is $k$-fat above $\left.\operatorname{root}(p)\right\}$ is dense in $\mathbb{P}$ for every $k \in \omega$.

Corollary 3.16 $\{p \in \mathbb{P}:|\operatorname{root}(p)| \geq k\}$ is dense in $\mathbb{P}$ for every $k \in \omega$.

Next we describe how $\mathbb{P}$ adds a generic real. The last corollary easily implies that if $G \subset \mathbb{P}$ is a generic filter then $\dot{x}_{G}=\bigcup_{p \in G} \operatorname{root}(p)$ is a function $\dot{x}_{G} \in \prod_{m \in \omega} \mathbb{Z}_{m+3}$. From now on we denote by $\dot{x}$ a name for this generic real.

Remark 3.17 Some textbooks require that forcing posets have largest elements, but our $\mathbb{P}$ has no such element. One possible answer to this problem is that one can actually do forcing without largest elements (since we can basically 'add a largest element to $\mathbb{P}^{\prime}$ ), and hence some other textbooks actually avoid largest elements in the definition of forcing posets. But there is another possible answer in case of $\mathbb{P}$; by mimicking the proof of Lemma 3.12 it is not hard to see that $\mathbb{P}$ is dense in

$$
\mathbb{P}_{0}=\left\{p \subset \Sigma: \forall k \in \omega \forall^{\infty} t \in p p[t] \text { is } k \text {-fat above } t\right\},
$$

which already has a largest element, namely $\Sigma$.

The reason why we prefer $\mathbb{P}$ to the apparently simpler $\mathbb{P}_{0}$ is that it fits our fusion arguments (inductive constructions) better.

\subsection{Properness and preservation of non-meagreness}

It will be necessary, of course, to prove that $\mathbb{P}$ is proper, but for the intended iteration we will need a stronger property. Recall that $p^{\prime} \in \mathbb{P}$ is $\mathfrak{M}$-generic, if for every dense open $D \subset \mathbb{P}$ if $D \in \mathfrak{M}$ then $p^{\prime} \Vdash$ " $D \cap \dot{G} \cap \mathfrak{M} \neq \emptyset$ ". (Here $\dot{G}$ is a name for the generic filter.) 
Definition 3.18 A forcing notion $\mathbb{P}$ is said to be Cohen-preserving if for every condition $p \in \mathbb{P}$, every countable elementary submodel $\mathfrak{M}$ such that $p, \mathbb{P}, \leq_{\mathbb{P}} \in \mathfrak{M}$ and every real $c$ that is a Cohen over $\mathfrak{M}$, there is an $\mathfrak{M}$-generic condition $p^{\prime} \leq p$ such that $p^{\prime} \Vdash " c$ is a Cohen real over $\mathfrak{M}[\dot{G}]$ ".

We now spell out this last clause in a bit more detail. For more information see e.g. [2]. Let $\mathcal{F}=\{f: \Sigma \rightarrow \Sigma \mid \forall \sigma \in \Sigma f(\sigma) \supset$ $\sigma\}$. Then the dense open subsets of $\prod_{m \in \omega} \mathbb{Z}_{m+3}$ are precisely the sets of the form $U_{f}=\bigcup_{\sigma \in \Sigma}[f(\sigma)]$, where $f$ ranges over $\mathcal{F}$. Then $p^{\prime} \Vdash$ " $c$ is a Cohen real over $\mathfrak{M}[\dot{G}]$ " means that if $\dot{f}$ is a name for an element of $\mathcal{F}$ and $\dot{f} \in \mathfrak{M}$ then $p^{\prime} \Vdash " c \in U_{\dot{f}} "$.

It is not hard to see that the notions of Cohen-preserving and second category set preserving partial order does not depend on the underlying Polish space. We will only use that $\prod_{m \in \omega} \mathbb{Z}_{m+3}$ and $\mathbb{R}$ are the same in this respect, which follows e.g. from the fact that we can throw away countable sets from these spaces so that the remaining sets are homeomorphic. (See the map in (1.1) in the discussion preceding Theorem 1.17.)

The following results are well-known, see e.g. [2].

Theorem 3.19 Cohen-preserving partial orders are proper and they preserve second category sets. The countable support iteration of Cohenpreserving partial orders is also Cohen-preserving.

Lemma 3.20 Let $p^{*} \in \mathbb{P}, \dot{f}^{*}$ be a name for an element of $\mathcal{F}$ and $D^{*}$ be a dense open subset of $\mathbb{P}$ such that $p^{*}, \dot{f}^{*}, D^{*} \in \mathfrak{M}$. Then there exists $q^{*} \leq p^{*}$ such that $q^{*} \in \mathfrak{M} \cap D^{*}$ and $q^{*} \Vdash$ "c $c \in U_{\dot{f}^{*}}$ ".

Proof. Define

$$
V=\bigcup\left\{\left[\sigma^{\prime}\right]: \exists q^{*} \leq p^{*} \exists \sigma \in \Sigma q^{*} \Vdash “ \dot{f}^{*}(\sigma)=\sigma^{\prime} "\right\} .
$$

It is not hard to see that $V \subset \prod_{m \in \omega} \mathbb{Z}_{m+3}$ is dense open and $V \in \mathfrak{M}$. Since $c$ is Cohen over $\mathfrak{M}$, we obtain $c \in V$, so we can find $q^{*}, \sigma$ and $\sigma^{\prime}$ such that $c \in\left[\sigma^{\prime}\right]$ and $q^{*} \Vdash " \dot{f}^{*}(\sigma)=\sigma^{\prime} "$. Let us now fix $\sigma$ and $\sigma^{\prime}$, then clearly $\exists q^{*} \in D^{*} q^{*} \leq p^{*} q^{*} \Vdash " \dot{f}^{*}(\sigma)=\sigma^{\prime} "$. Applying elementarity to this last formula we obtain such a $q^{*} \in \mathfrak{M}$. Since $q^{*} \Vdash$ " $\dot{f}^{*}(\sigma)=\sigma^{\prime}$ " clearly implies $q^{*} \Vdash$ "c $\in U_{\dot{f}}$ ", the proof is complete.

Now we are ready to prove the main result of the section. The proof will essentially be an inductive construction of a condition. Unlike in the proof of 
Lemma 3.12, we will not build the tree 'level-by level', but we will perform a kind of 'back-and forth' fusion instead. The only place where this more complicated fusion is essential is Lemma 3.34, but we decided to use this method here in a simpler situation as well to make the reading of Lemma 3.34 easier.

Lemma 3.21 $\mathbb{P}$ is Cohen-preserving (and hence proper, as well).

Proof. Let $\left\{\dot{f}_{n}\right\}_{n \in \omega}$ enumerate the names for elements of $\mathcal{F}$ that are in $\mathfrak{M}$, and let $\left\{D_{n}\right\}_{n \in \omega}$ enumerate the dense open subsets of $\mathbb{P}$ that are in $\mathfrak{M}$.

For $n \in \omega$ we will inductively define

(i) $s_{n} \in \Sigma$,

(ii) $q_{n} \in \mathbb{P}$,

(iii) $t_{n} \in \Sigma$,

(iv) $p_{n} \in \mathbb{P}$,

such that for every $m \leq n$ the following hold:

(1) $t_{m} \in p_{n}$,

(2) $\operatorname{succ}_{p_{m}}\left(t_{m}\right) \backslash\left\{s_{0}, \ldots, s_{n}\right\} \subset \operatorname{succ}_{p_{n}}\left(t_{m}\right)$,

(3) $\operatorname{succ}_{p_{m}}\left(t_{m}\right)$ is $(m+1)$-fat above $t_{m}$,

(4) $p \geq p_{0}$ and $p_{m} \geq p_{n}$,

(5) $q_{m}=p_{m}\left[t_{m}\right]$

(6) $q_{n} \in D_{m}$,

(7) $q_{n} \Vdash " c \in U_{f_{m}^{\prime}} "$.

We will make sure that every stage of the induction will be carried out in $\mathfrak{M}$, and we will tacitly assume that all object we pick at the stages are in $\mathfrak{M}$. (The whole induction will of course not be in $\mathfrak{M}$.)

Let us start with $n=0$. Put $s_{0}=\emptyset$. By Lemma 3.20. Corollary 3.15 and Fact 3.13 there exists $q_{0} \leq p$ such that $q_{0} \in D_{0}, q_{0} \Vdash$ "c $\in U_{\dot{f}_{0}}$ ", and 
if $t_{0}=\operatorname{root}\left(q_{0}\right)$ then $\operatorname{succ}_{q_{0}}\left(t_{0}\right)$ is 1 -fat above $t_{0}$. Setting $p_{0}=q_{0}$ finishes the $0^{\text {th }}$ step. It is not hard to check that the inductive assumptions are satisfied.

Let us now assume that $s_{m}, q_{m}, t_{m}$ and $p_{m}$ have already been defined for $m \leq n$ satisfying the inductive assumptions. For every $m$ let $\left\{S_{m}^{k}\right\}_{k \in \omega}$ be an enumeration of the set of $(m+1)$-slaloms above $t_{m}$. To start the $n+1^{\text {st }}$ step, first we need to pick a $t_{m}$ for some $m \leq n$. We make sure by some simple bookkeeping that during the course of the induction each $t_{m}$ will be picked infinitely many times, and when we visit the node $t_{m}$ for the $k^{t h}$ time then we take care of $S_{m}^{k}$ (we construct a $t_{n+1}$ above $t_{m}$ escaping $S_{m}^{k}$ ).

So let us assume that we are at the $n+1^{\text {st }}$ step and we pick $t_{m}$ for the $k^{\text {th }}$ time. Inductive assumption (3) yields that $\operatorname{succ}_{p_{m}}\left(t_{m}\right)$ is $(m+1)$-fat above $t_{m}$, hence so is $\operatorname{succ}_{p_{m}}\left(t_{m}\right) \backslash\left\{s_{0}, \ldots, s_{n}\right\}$ by Fact 3.7. Thus we can fix an $s_{n+1} \in \operatorname{succ}_{p_{m}}\left(t_{m}\right) \backslash\left\{s_{0}, \ldots, s_{n}\right\}$ escaping the $(m+1)$-slalom $S_{m}^{k}$. By (2) we have $s_{n+1} \in p_{n}$ as well. Applying Lemma $3.20 n+2$ times we obtain a $q_{n+1} \leq$ $p_{n}\left[s_{n+1}\right]$ such that $q_{n+1} \in \bigcap_{m \leq n+1} D_{m}$ and $q_{n+1} \Vdash " c \in \bigcap_{m \leq n+1} U_{\dot{f_{m}}}$ ". By Corollary 3.15 and Fact 3.13 we may assume that if $t_{n+1}=\operatorname{root}\left(q_{n+1}\right)$ then $\operatorname{succ}_{q_{n+1}}\left(t_{n+1}\right)$ is $n+2$-fat above $t_{n+1}$. Setting $p_{n+1}=\left(p_{n} \backslash p_{n}\left[s_{n+1}\right]\right) \cup q_{n+1}$ finishes the $n+1^{\text {st }}$ step.

Now we check that the inductive assumptions are satisfied. Items (1) and (2) follow from the structure of the fusion. Namely, it is not hard to see that at the $n+1^{\text {st }}$ step we only modify $p_{n}$ in the 'cone' $p_{n}\left[s_{n+1}\right]$, and this cone does not contain the earlier $t_{m}$ 's, moreover, an element of $\operatorname{succ}_{p_{m}}\left(t_{m}\right)$ only 'disappears' from $p_{n}$ when it is picked as an $s_{n+1}$. Items (44), (5), (6) and (7) are straightforward from the construction, and (3) follows from (5).

Let us now define $p^{\prime}=\left\{t_{m}\right\}_{m \in \omega}$. It is easy to see that $p^{\prime} \in \mathbb{P}$, since we made sure by the bookkeeping that $\operatorname{succ}_{p^{\prime}}\left(t_{m}\right)$ is $m+1$-fat above $t_{m}$ for every $m$. Combining (11) and (4) we obtain

$$
p^{\prime} \leq p_{n}
$$

for every $n$, and also that $p^{\prime} \leq p$.

All that remains to be shown is that $p^{\prime}$ is $\mathfrak{M}$-generic and $p^{\prime} \Vdash$ "c is a Cohen real over $\mathfrak{M}[\dot{G}] "$.

First we check that $p^{\prime}$ is $\mathfrak{M}$-generic. Let $n_{0} \in \omega$ be fixed, then we have to show that $p^{\prime} \Vdash$ " $D_{n_{0}} \cap \dot{G} \cap \mathfrak{M} \neq \emptyset$ ". Let $p^{\prime \prime} \leq p^{\prime}$ be arbitrary, it suffices to find a $p^{\prime \prime \prime} \leq p^{\prime \prime}$ such that $p^{\prime \prime \prime} \Vdash$ " $D_{n_{0}} \cap \dot{G} \cap \mathfrak{M} \neq \emptyset$ ". Since every condition is infinite, there exists $n \geq n_{0}$ such that $t_{n} \in p^{\prime \prime}$. By (6) we have $q_{n} \in D_{n_{0}}$ and also $q_{n} \in \mathfrak{M}$, so $q_{n} \Vdash$ " $D_{n_{0}} \cap \dot{G} \cap \mathfrak{M} \neq \emptyset$ ". Thus, (3.1) and (15) imply $p^{\prime \prime}\left[t_{n}\right] \leq p^{\prime}\left[t_{n}\right] \leq p_{n}\left[t_{n}\right]=q_{n}$, so we are done by choosing $p^{\prime \prime \prime}=p^{\prime \prime}\left[t_{n}\right]$. 
A similar argument shows that $p^{\prime} \Vdash " c$ is a Cohen real over $\mathfrak{M}[\dot{G}]$ ". Indeed, for every $n_{0}$ and $p^{\prime \prime} \leq p^{\prime}$ there exists $n \geq n_{0}$ such that $t_{n} \in p^{\prime \prime}$. Then $q_{n} \Vdash " c \in U_{\dot{f}_{n_{0}}}$ ", $q_{n} \in \mathfrak{M}$, and $p^{\prime \prime \prime}=p^{\prime \prime}\left[t_{n}\right] \leq p^{\prime}\left[t_{n}\right] \leq p_{n}\left[t_{n}\right]=q_{n}$, so we are done. This finishes the proof of the lemma.

\subsection{The Main Lemma}

Our main lemma will describe how a single step in the iterated forcing construction works.

Lemma 3.22 Let $X \in V$ such that $X \subset \prod_{m \in \omega} \mathbb{Z}_{m+3}$ and $X$ is non-meagre in every non-empty open subset of $\prod_{m \in \omega} \mathbb{Z}_{m+3}$. Then $\mathbb{P} \Vdash$ " $X \cap\left(\dot{C}_{E K}-\right.$ $\dot{x})$ is non-meagre in $\dot{C}_{E K}-\dot{x}$.

Proof. It is not hard to see that if a set $H \subset C_{E K}-x$ is meagre in $C_{E K}-x$ then there exists a decreasing sequence of open sets $U_{n} \subset \prod_{m \in \omega} \mathbb{Z}_{m+3}$ such that $U_{n} \subset B\left(C_{E K}-x, \frac{1}{n+1}\right), U_{n} \cap\left(C_{E K}-x\right)$ is dense in $C_{E K}-x$ and $\bigcap_{n} U_{n} \cap H=\emptyset$. (Here $B(A, \varepsilon)$ denotes the $\varepsilon$-neighbourhood of the set $A$.)

Hence let us assume that there exist $p \in \mathbb{P}$ and a name $\left\{\dot{U}_{n}\right\}_{n \in \omega}$ for a decreasing sequence of open subsets of $\prod_{m \in \omega} \mathbb{Z}_{m+3}$ such that $p \Vdash$ " $\dot{U}_{n} \subset$ $B\left(\dot{C}_{E K}-\dot{x}, \frac{1}{n+1}\right), \dot{U}_{n} \cap\left(\dot{C}_{E K}-\dot{x}\right)$ is dense in $\dot{C}_{E K}-\dot{x}$ and $\bigcap_{n} \dot{U}_{n} \cap X=\emptyset$ ".

Define

$$
R_{p,\left\{\dot{U}_{n}\right\}_{n \in \omega}}=\left\{r \in \prod_{m \in \omega} \mathbb{Z}_{m+3}: \exists p^{\prime} \leq p, p^{\prime} \Vdash " r \in \bigcap_{n} \dot{U}_{n} "\right\} .
$$

For the definitions and basic facts concerning analytic sets, sets with the property of Baire, etc, we refer the reader to [13].

In the next subsection we will prove that we can assume (by replacing $p$ with a stronger condition, if necessary) that $R_{p,\left\{\dot{U}_{n}\right\}_{n \in \omega}}$ is analytic. Therefore it possesses the property of Baire. Moreover, in Subsection 3.3.2 we will prove that $R_{p,\left\{\dot{U}_{n}\right\}_{n \in \omega}}$ is non-meagre. Let us now accept these statements for the moment. Then $R_{p,\left\{\dot{U}_{n}\right\}_{n \in \omega}}$ is co-meagre in a non-empty open set, thus $X \cap R_{p,\left\{\dot{U}_{n}\right\}_{n \in \omega}} \neq \emptyset$, so we can fix an $r \in X \cap R_{p,\left\{\dot{U}_{n}\right\}_{n \in \omega}}$. But then $r \in X$ and $p^{\prime} \Vdash$ " $r \in \bigcap_{n} \dot{U}_{n}$ " for some $p^{\prime} \leq p$, thus $p^{\prime} \Vdash " \bigcap_{n} \dot{U}_{n} \cap X \neq \emptyset$ ". On the other hand, $p^{\prime} \leq p$ implies that $p^{\prime} \Vdash$ " $\bigcap_{n} \dot{U}_{n} \cap X=\emptyset$ ", which is a contradiction.

Corollary 3.23 Let $X \in V, X \subset \prod_{m \in \omega} \mathbb{Z}_{m+3}, X$ is non-meagre. Then $\mathbb{P} \Vdash$ " $\exists t \in \prod_{m \in \omega} \mathbb{Z}_{m+3}$ such that $X \cap\left(\dot{C}_{E K}+t\right)$ is non-meagre in $\dot{C}_{E K}+t$ ". 
Proof. Let $Q$ be the analogue of the rationals, that is, $Q=\{q \in$ $\left.\prod_{m \in \omega} \mathbb{Z}_{m+3}: \forall^{\infty} m q(m)=0\right\}$. Then $X+Q$ is non-meagre in every non-empty open subset of $\prod_{m \in \omega} \mathbb{Z}_{m+3}$, hence $\mathbb{P} \Vdash$ " $(X+Q) \cap\left(\dot{C}_{E K}-\right.$ $\dot{x})$ is non-meagre in $\dot{C}_{E K}-\dot{x}$ " by the previous lemma. But $Q$ is countable and $X+Q=\bigcup_{q \in Q} X+q$, so $\mathbb{P} \Vdash " \exists q \in Q$ such that $(X+q) \cap$ $\left(\dot{C}_{E K}-\dot{x}\right)$ is non-meagre in $\dot{C}_{E K}-\dot{x}$ ". But then $\mathbb{P} \Vdash$ " $X \cap\left(\dot{C}_{E K}-\dot{x}-\right.$ $q$ ) is non-meagre in $\dot{C}_{E K}-\dot{x}-q$ " and we are done.

We still have to prove the two statements concerning $R_{p,\left\{\dot{U}_{n}\right\}_{n \in \omega}}$.

\subsubsection{Analyticity of $R_{p,\left\{\dot{U}_{n}\right\}_{n \in \omega}}$}

As above, let $p \in \mathbb{P}$ and $\left\{\dot{U}_{n}\right\}_{n \in \omega}$ be a name for a decreasing sequence of open sets of $\prod_{m \in \omega} \mathbb{Z}_{m+3}$ such that $p \Vdash$ " $\dot{U}_{n} \subset B\left(\dot{C}_{E K}-\dot{x}, \frac{1}{n+1}\right), \dot{U}_{n} \cap\left(\dot{C}_{E K}-\dot{x}\right)$ is dense in $\dot{C}_{E K}-\dot{x}$ and $\bigcap_{n} \dot{U}_{n} \cap X=\emptyset "$. Recall that

$$
R_{p,\left\{\dot{U}_{n}\right\}_{n \in \omega}}=\left\{r \in \prod_{m \in \omega} \mathbb{Z}_{m+3}: \exists p^{\prime} \leq p, p^{\prime} \Vdash " r \in \bigcap_{n} \dot{U}_{n} "\right\} .
$$

Lemma 3.24 There exists a $q \leq p$ such that $R_{q,\left\{\dot{U}_{n}\right\}_{n \in \omega}}$ is analytic.

We will split the proof into several steps.

Definition 3.25 Let $p \in \mathbb{P}$. A set $B \subset p$ is called a barrier if it intersects every infinite branch of $p$. It is open, if $s, t \in p, s \subset t, s \in B$ imply $t \in B$.

Definition 3.26 Let $p$ and $\left\{\dot{U}_{n}\right\}_{n \in \omega}$ be as above. Then $p$ is nice with respect to $\left\{\dot{U}_{n}\right\}_{n \in \omega}$ if for every $s \in \Sigma$ and every $n \in \omega$

$$
B_{s, n}=\left\{t \in p: p[t] \Vdash “[s] \subset \dot{U}_{n} \text { or } p[t] " \Vdash[s] \not \subset \dot{U}_{n} "\right\}
$$

is a barrier. (It is clearly open.)

Note that if $B \subset p$ is an open barrier and $q \leq p$ then $q \cap B \neq \emptyset$.

Lemma 3.27 Assume that $p$ is nice with respect to $\left\{\dot{U}_{n}\right\}_{n \in \omega}$. Let $q^{*} \leq p$, $n^{*} \in \omega, r \in \prod_{m \in \omega} \mathbb{Z}_{m+3}$ and $q^{*} \Vdash$ " $r \in \dot{U}_{n^{*}}$ ". Then there are $t^{*} \in q^{*}$ and $k^{*} \in \omega$ such that $p\left[t^{*}\right] \Vdash "\left[r \mid k^{*}\right] \subset \dot{U}_{n^{*}}$. 
Proof. There are $q^{\prime} \leq q^{*}$ and $k^{*} \in \omega$ such that $q^{\prime} \Vdash "\left[r \mid k^{*}\right] \subset \dot{U}_{n^{*}}$ ". Since $B_{r \mid k^{*}, n^{*}}$ is an open barrier in $p$ and $q^{\prime} \leq p$, we obtain that there is a $t^{*} \in q^{\prime} \cap B_{r \mid k^{*}, n^{*}}$. Then $p\left[t^{*}\right] \Vdash$ " $\left[r \mid k^{*}\right] \not \subset \dot{U}_{n^{*}}$ " is impossible, since then $q^{\prime}\left[t^{*}\right] \leq p\left[t^{*}\right], q^{\prime}$ would force two contradicting statements. Hence $p\left[t^{*}\right] \Vdash$ " $\left[r \mid k^{*}\right] \subset \dot{U}_{n^{*}}$ " by the definition of $B_{r \mid k^{*}, n^{*}}$, and we are done.

Lemma 3.28 Assume that $p$ is nice with respect to $\left\{\dot{U}_{n}\right\}_{n \in \omega}$. Let $r \in$ $\prod_{m \in \omega} \mathbb{Z}_{m+3}$ be arbitrary. Then $r \in R_{p,\left\{\dot{U}_{n}\right\}_{n \in \omega}}$ iff there exists a sequence of sets $B_{n} \subset p$ and a sequence of functions $\varphi_{n}: B_{n} \rightarrow \omega$ such that for every $n \in \omega$

(1) $\left|B_{0}\right|=1$

(2) $B_{n}$ consists of pairwise incomparable sequences,

(3) $\forall t \in B_{n+1} \exists s \in B_{n} s \varsubsetneqq t$,

(4) $\forall s \in B_{n} \varphi_{n}(s)>n$,

(5) $\forall k \in \omega \forall^{\infty} s \in B_{n} \varphi_{n}(s) \geq k$,

(6) $\forall s \in B_{n}\left\{t \in B_{n+1}: t \supset s\right\}$ is $\varphi_{n}(s)$-fat above $s$,

(7) $\forall t \in B_{n} \exists k \in \omega p[t] \Vdash "[r \mid k] \subset \dot{U}_{n} "$.

Proof. Let us first suppose $r \in R_{p,\left\{\dot{U}_{n}\right\}_{n \in \omega}}$, that is, $p^{\prime} \Vdash$ " $r \in \bigcap_{n} \dot{U}_{n}$ " for some $p^{\prime} \leq p$. We construct $\left\{B_{n}\right\}_{n \in \omega}$ and $\left\{\varphi_{n}\right\}_{n \in \omega}$ by induction on $n$. We will make the induction hypothesis that

$$
\forall s \in B_{n} \operatorname{succ}_{p^{\prime}}(s) \text { is } \varphi_{n}(s) \text {-fat above } s \text {. }
$$

Applying the previous lemma with $q^{*}=p^{\prime}$ and $n^{*}=0$ we obtain $t_{0} \in p^{\prime}$ and $k_{0} \in \omega$ such that $p\left[t_{0}\right] \Vdash "\left[r \mid k_{0}\right] \subset \dot{U}_{0} "$. Put $B_{0}=\left\{t_{0}\right\}$. By Fact 3.10 we may assume (by extending $t_{0}$ in $p^{\prime}$ if necessary) that $\operatorname{succ}_{p^{\prime}}\left(t_{0}\right)$ is 1 -fat above $t_{0}$. Define $\varphi_{0}\left(t_{0}\right)=1$. Then all requirements imposed on $B_{0}$ and $\varphi_{0}$ are satisfied.

Suppose that $B_{n}$ and $\varphi_{n}$ satisfying all requirements have already been constructed. For each $t \in \bigcup_{s \in B_{n}} \operatorname{succ}_{p^{\prime}}(s)$ let us apply the previous lemma with $q^{*}=p^{\prime}[t]$ and $n^{*}=n+1$. Thus we obtain $u_{t} \in p^{\prime}, u_{t} \supset t$ and $k_{t} \in \omega$ such 
that $p\left[u_{t}\right] \Vdash "\left[r \mid k_{t}\right] \subset \dot{U}_{n+1} "$. Let $\psi: \bigcup_{s \in B_{n}} \operatorname{succ}_{p^{\prime}}(s) \rightarrow \omega$ be an arbitrary function satisfying

$$
\forall t \in \bigcup_{s \in B_{n}} \operatorname{succ}_{p^{\prime}}(s) \quad \psi(t)>n+1
$$

and

$$
\forall k \in \omega \forall^{\infty} t \in \bigcup_{s \in B_{n}} \operatorname{succ}_{p^{\prime}}(s) \quad \psi(t) \geq k .
$$

Using Fact 3.10 we may assume that $u_{t}$ is $\psi(t)$-fat above $u_{t}$ for every $t$. (Note that extending $u_{t}$ in $p^{\prime}$ if necessary does not harm any of the requirements.) Then putting $B_{n+1}=\left\{u_{t}: t \in \bigcup_{s \in B_{n}} \operatorname{succ}_{p^{\prime}}(s)\right\}$ and $\varphi_{n+1}\left(u_{t}\right)=\psi(t)$ finishes the proof of this direction.

In order to prove the other direction, let us assume that $\left\{B_{n}\right\}_{n \in \omega}$ and $\left\{\varphi_{n}\right\}_{n \in \omega}$ satisfy all requirements. Set $p^{\prime}=\bigcup_{n} B_{n}$. It is easy to see that $p^{\prime} \in \mathbb{P}$ and $p^{\prime} \leq p$. Therefore it suffices to check that for every $n_{0} \in \omega$ we have $p^{\prime} \Vdash$ " $r \in \dot{U}_{n_{0}}$ ". Otherwise, there exists a $p^{\prime \prime} \leq p^{\prime}$ such that

$$
p^{\prime \prime} \Vdash " r \notin \dot{U}_{n_{0}} " .
$$

It is easy to see from the construction of $p^{\prime}$ that $B_{n_{0}}^{\prime}=\left\{s \in p^{\prime}: \exists t \in B_{n_{0}} \quad t \subset\right.$ $s\}$ is an open barrier in $p^{\prime}$, hence $p^{\prime \prime} \cap B_{n_{0}}^{\prime} \neq \emptyset$. Thus there are $t \in B_{n_{0}}$ and $s \supset t$ with $s \in p^{\prime \prime}$. Then $p^{\prime \prime}[s] \leq p[t], p^{\prime \prime}$, and $p[t] \Vdash " r \in \dot{U}_{n_{0}}$ " by (7), which is a contradiction by (3.2).

Lemma 3.29 Let $p$ and $\left\{\dot{U}_{n}\right\}_{n \in \omega}$ be as above. Then there exists $q \leq p$ that is nice with respect to $\left\{\dot{U}_{n}\right\}_{n \in \omega}$.

Proof. The proof will be similar to the previous inductive construction, so we will omit some details. Let $\left\{\left(s_{i}, n_{i}\right)\right\}_{i \in \omega}$ be an enumeration of $\Sigma \times \omega$. We inductively define a sequence $B_{i} \subset p$ and for every $t \in B_{i}$ a condition $p_{t} \leq p$ with $\operatorname{root}\left(p_{t}\right)=t$ as follows.

The fact ' $q \Vdash \varphi$ or $q \Vdash \neg \varphi$ ' will be abbreviated as $q \| \varphi$.

There exists $p^{\prime} \leq p$ such that $p^{\prime} \|$ " $\left[s_{0}\right] \subset \dot{U}_{n_{0}}$ ". We may assume that $\operatorname{succ}_{p^{\prime}}\left(\operatorname{root}\left(p^{\prime}\right)\right)$ is 1-fat above $\operatorname{root}\left(p^{\prime}\right)$. Let $t_{0}=\operatorname{root}\left(p^{\prime}\right)$ and put $B_{0}=\left\{t_{0}\right\}$, $p_{t_{0}}=p^{\prime}$.

Now assume that $B_{i}$ and $p_{t}$ for every $t \in B_{i}$ have already been constructed. For every $t \in B_{i}$ and every $u \in \operatorname{succ}_{p_{t}}(t)$ find $p^{\prime} \leq p_{t}[u]$ such that 
$p^{\prime} \|$ " $\left[s_{i+1}\right] \subset \dot{U}_{n_{i+1}}$ " and $\operatorname{succ}_{p^{\prime}}\left(\operatorname{root}\left(p^{\prime}\right)\right)$ is 'sufficiently fat above $\operatorname{root}\left(p^{\prime}\right)$ '. Then let $B_{i+1}$ be the set of these $\operatorname{root}\left(p^{\prime}\right)$ 's and let $p_{\operatorname{root}\left(p^{\prime}\right)}=p^{\prime}$. This finishes the general step of the induction. Note that $p_{t} \|{ }^{\prime \prime}\left[s_{i}\right] \subset \dot{U}_{n_{i}}$ " for every $i \in \omega$ and every $t \in B_{i}$.

Then it is not hard to see that $q=\bigcup_{i} B_{i} \in \mathbb{P}$ and $q \leq p$. It easily follows from the construction that every $B_{i}$ is a barrier in $q$. Let us now check that $q$ is nice with respect to $\left\{\dot{U}_{n}\right\}_{n \in \omega}$. So let us fix $i \in \omega$, and it suffices to show that $q[t] \| "\left[s_{i}\right] \subset \dot{U}_{n_{i}}$ " for every $t \in B_{i}$. But this is clear, since $p_{t} \|$ " $\left[s_{i}\right] \subset \dot{U}_{n_{i}}$ " and $q[t] \leq p_{t}$.

Now we are ready to prove Lemma 3.24 .

Proof. By the previous lemma we may assume that $p$ is nice with respect to $\left\{\dot{U}_{n}\right\}_{n \in \omega}$. Define

$$
\begin{gathered}
B_{p,\left\{\dot{U}_{n}\right\}_{n \in \omega}}= \\
\left\{\left(r,\left\{B_{n}\right\}_{n \in \omega},\left\{\varphi_{n}\right\}_{n \in \omega}\right): r,\left\{B_{n}\right\}_{n \in \omega} \text { and }\left\{\varphi_{n}\right\}_{n \in \omega} \text { are as in Lemma 3.28 }\right\} \\
\subset \prod_{m \in \omega} \mathbb{Z}_{m+3} \times\left(2^{p}\right)^{\omega} \times\left(2^{p \times \omega}\right)^{\omega},
\end{gathered}
$$

where $\mathbb{Z}_{m+3}, \omega$ and $p$ are all equipped with the discrete topology, hence this huge ambient product space is compact metric, therefore Polish. It suffices to prove that $B_{p,\left\{\dot{U}_{n}\right\}_{n \in \omega}}$ is a Borel set in this product space, since then $R_{p,\left\{\dot{U}_{n}\right\}_{n \in \omega}}$, which is the projection of $B_{p,\left\{\dot{U}_{n}\right\}_{n \in \omega}}$ on the first coordinate, is analytic. We mostly leave this standard but very lengthy computation to the reader, and only deal with the most interesting clause, that is, Lemma 3.28 (7).

The conditions ' $\forall n \in \omega$ ', ' $\forall t \in p$ ', ' $t \in B_{n}$ ', ' $\exists k \in \omega$ ' are clearly Borel, so it suffices to check that for fixed $n, t$ and $k$

$$
V_{n, t, k}=\left\{r \in \prod_{m \in \omega} \mathbb{Z}_{m+3}: p[t] \Vdash "[r \mid k] \subset \dot{U}_{n} "\right\}
$$

is Borel. But clearly

$$
V_{n, t, k}=\bigcup\left\{[s]: s \in \Sigma,|s|=k, p[t] \Vdash "[s] \subset \dot{U}_{n} "\right\},
$$

so it is actually a union of basic clopen sets, hence open. 


\subsubsection{Non-meagreness of $R_{p,\left\{\dot{U}_{n}\right\}_{n \in \omega}}$}

Yet again, let $p \in \mathbb{P}$ and $\left\{\dot{U}_{n}\right\}_{n \in \omega}$ be a name for a decreasing sequence of open sets of $\prod_{m \in \omega} \mathbb{Z}_{m+3}$ such that $p \Vdash$ " $\dot{U}_{n} \subset B\left(\dot{C}_{E K}-\dot{x}, \frac{1}{n+1}\right), \dot{U}_{n} \cap\left(\dot{C}_{E K}-\dot{x}\right)$ is dense in $\dot{C}_{E K}-\dot{x}$ and $\bigcap_{n} \dot{U}_{n} \cap X=\emptyset "$. Recall that

$$
R_{p,\left\{\dot{U}_{n}\right\}_{n \in \omega}}=\left\{r \in \prod_{m \in \omega} \mathbb{Z}_{m+3}: \exists q \leq p, q \Vdash " r \in \bigcap_{n} \dot{U}_{n} "\right\} .
$$

Lemma 3.30 Let $q^{*} \leq p, s^{*} \in \Sigma$ and $l^{*} \in \omega$ such that $q^{*} \Vdash "\left[s^{*}\right] \cap\left(\dot{C}_{E K}-\right.$ $\dot{x}) \neq \emptyset "$. Then there exist $q^{* *} \leq q^{*}$ and $s^{* *} \in \Sigma, s^{* *} \supset s^{*}$ such that $q^{* *} \Vdash$ " $\left[s^{* *}\right] \cap\left(\dot{C}_{E K}-\dot{x}\right) \neq \emptyset$ and $\left[s^{* *}\right] \subset \dot{U}_{l^{*}} "$.

Proof. Set $\dot{C}=\dot{C}_{E K}-\dot{x}$. Since $p \Vdash$ " $\dot{U}_{l^{*}} \cap \dot{C}$ is dense open in $\dot{C}$ " and $q^{*} \Vdash$ " $\left[s^{*}\right] \cap \dot{C}$ is non-empty open in $\dot{C}$ " we obtain that $q^{*} \Vdash "\left[s^{*}\right] \cap \dot{U}_{l^{*}} \cap \dot{C} \neq \emptyset$ ". Since $\left[s^{*}\right] \cap \dot{U}_{l^{*}}$ is a name for an open subset of $\prod_{m \in \omega} \mathbb{Z}_{m+3}$, and if an open set meets a set then it contains a basic open set meeting the same set, we infer that $q^{*} \Vdash$ " $\exists \dot{s}^{* *} \in \Sigma,\left[\dot{s}^{* *}\right] \cap \dot{C} \neq \emptyset,\left[\dot{s}^{* *}\right] \subset\left[s^{*}\right] \cap \dot{U}_{l^{*}}$ ". Hence there exist $q^{* *} \leq q^{*}$ and $s^{* *} \in \Sigma, s^{* *} \supset s^{*}$ such that $q^{* *} \Vdash "\left[s^{* *}\right] \cap \dot{C} \neq \emptyset$ and $\left[s^{* *}\right] \subset \dot{U}_{l^{*}}$.

Definition 3.31 We will write $s \| t$ to denote that $s(i)+t(i) \neq i+2$ for every $i<\min (|s|,|t|)$.

For $p \in \mathbb{P}$ and $t \in \Sigma$ the symbol $p \| t$ will abbreviate that $s \| t$ for every $s \in p$.

The following fact can be easily checked by a standard argument using that $p \Vdash \varphi \Longleftrightarrow \forall p^{\prime} \leq p \exists p^{\prime \prime} \leq p^{\prime} p^{\prime \prime} \Vdash \varphi$. The details are left to the reader.

Fact 3.32 Let $p \in \mathbb{P}$ and $t \in \Sigma$. Then the following are equivalent.

1. $p \| t$,

2. $p \Vdash "[t] \cap\left(\dot{C}_{E K}-\dot{x}\right) \neq \emptyset "$.

We will also need one more lemma. The proof, which is left to the reader again, follows easily from the definition of fatness.

Lemma 3.33 Let $k>0, s_{0} \in \Sigma, F \subset\left\{s \in \Sigma: s \supset s_{0}\right\}$ be $k$-fat above $s_{0}$, and also let $\sigma \in \Sigma$ such that $s_{0} \| \sigma$. Then $\{t \in F: t \| \sigma\}$ is $k-1$-fat above $s_{0}$. 
We are now ready to prove what we are aiming at.

Lemma 3.34 $R_{p,\left\{\dot{U}_{n}\right\}_{n \in \omega}}$ is non-meagre.

Proof. We have to show that $R_{p,\left\{\dot{U}_{n}\right\}_{n \in \omega}}$ intersects every dense $G_{\delta}$ set, so it suffices to prove that $R_{p,\left\{\dot{U}_{n}\right\}_{n \in \omega}} \cap \bigcap_{n} V_{n}=\emptyset$ for every sequence $\left\{V_{n}\right\}_{n \in \omega}$ of dense open subsets of $\prod_{m \in \omega} \mathbb{Z}_{m+3}$. The proof will work as follows. On the one hand, we will inductively define a strictly increasing sequence $\left\{r_{n}\right\}_{n \in \omega}$ of elements of $\Sigma$ such that $\left[r_{n}\right] \subset V_{n}$, which will of course imply that if we set $r=\bigcup_{n} r_{n}$ then $r \in \bigcap_{n} V_{n}$. On the other hand, we will also simultaneously carry out a fusion argument similar to the one in the proof of Lemma 3.21 and obtain a $p^{\prime} \leq p$ such that $p^{\prime} \Vdash$ " $r \in \bigcap_{n} \dot{U}_{n}$ ". This will show $r \in$ $R_{p,\left\{\dot{U}_{n}\right\}_{n \in \omega}} \cap \bigcap_{n} V_{n}$, which will complete the proof.

Let us now start the fusion. The main differences between this argument and the one in Lemma 3.21 will be that we will use Lemma 3.30 instead of Lemma 3.20, and we will be building the $r_{n}$ 's as well.

For $n \in \omega$ we will inductively define

(i) $s_{n} \in \Sigma$,

(ii) $q_{n} \in \mathbb{P}$,

(iii) $t_{n} \in \Sigma$,

(iv) $r_{n} \in \Sigma$,

(v) $p_{n} \in \mathbb{P}$,

such that for every $m \leq n$ the following hold:

(1) $r_{n} \| t_{m}$,

(2) $\left|r_{n}\right| \geq\left|t_{m}\right|$,

(3) $t_{m} \in p_{n}$,

(4) $\operatorname{succ}_{p_{m}}\left(t_{m}\right) \backslash\left\{s_{0}, \ldots, s_{n}\right\} \subset \operatorname{succ}_{p_{n}}\left(t_{m}\right)$,

(5) $\operatorname{succ}_{p_{m}}\left(t_{m}\right)$ is $(m+2)$-fat above $t_{m}$,

(6) $p \geq p_{0}$ and $p_{m} \geq p_{n}$, 
(7) $q_{m}=p_{m}\left[t_{m}\right]$

(8) $q_{m} \Vdash "\left[r_{m}\right] \subset \dot{U}_{m} "$.

Let us start with $n=0$. Put $s_{0}=\emptyset$. Applying Lemma 3.30 to $p, \emptyset$ and 0, then using Corollary 3.15 and Fact 3.13, we obtain that there exists $q_{0} \leq p$ and $r_{0}^{\prime} \in \Sigma$ such that $q_{0} \Vdash " ~ "\left[r_{0}^{\prime}\right] \cap\left(\dot{C}_{E K}-\dot{x}\right) \neq \emptyset$ and $\left[r_{0}^{\prime}\right] \subset \dot{U}_{0} "$, and if $t_{0}=\operatorname{root}\left(q_{0}\right)$ then $\operatorname{succ}_{q_{0}}\left(t_{0}\right)$ is 2 -fat above $t_{0}$. Then $r_{0}^{\prime} \| t_{0}$ by Fact 3.32 . Hence we can clearly find an $r_{0}^{\prime \prime} \supset r_{0}^{\prime}$ such that $\left|r_{0}^{\prime \prime}\right| \geq\left|t_{0}\right|$ and $r_{0}^{\prime \prime}|| t_{0}$. Finally, since $V_{0}$ is dense open, we can extend $r_{0}^{\prime \prime}$ further to obtain an $r_{0} \supset r_{0}^{\prime \prime}$ with $\left[r_{0}\right] \subset V_{0}$. Setting $p_{0}=q_{0}$ finishes the $0^{t h}$ step. It is not hard to check that the inductive assumptions are satisfied. (Note that (11) and (2) follow from $\left|r_{0}^{\prime \prime}\right| \geq\left|t_{0}\right|, r_{0}^{\prime \prime}|| t_{0}$, and $\left.r_{0} \supset r_{0}^{\prime \prime}.\right)$

Let us now assume that $s_{m}, q_{m}, t_{m}, r_{m}$ and $p_{m}$ have already been defined for $m \leq n$ satisfying the inductive assumptions. For every $m$ let $\left\{S_{m}^{k}\right\}_{k \in \omega}$ be an enumeration of the set of $(m+1)$-slaloms above $t_{m}$. To start the $n+1^{\text {st }}$ step, first we need to pick a $t_{m}$ for some $m \leq n$. We make sure by some simple bookkeeping that during the course of the induction each $t_{m}$ will be picked infinitely many times, and when we visit the node $t_{m}$ for the $k^{\text {th }}$ time then we take care of $S_{m}^{k}$ (we construct a $t_{n+1}$ above $t_{m}$ escaping $S_{m}^{k}$ ).

So let us assume that we are at the $n+1^{\text {st }}$ step and we pick $t_{m}$ for the $k^{\text {th }}$ time. Inductive assumption (5) yields that $\operatorname{succ}_{p_{m}}\left(t_{m}\right)$ is $(m+2)$-fat above $t_{m}$, hence so is $\operatorname{succ}_{p_{m}}\left(t_{m}\right) \backslash\left\{s_{0}, \ldots, s_{n}\right\}$ by Fact 3.7. Therefore $\left\{t \in \operatorname{succ}_{p_{m}}\left(t_{m}\right) \backslash\right.$ $\left.\left\{s_{0}, \ldots, s_{n}\right\}: t \| r_{n}\right\}$ is $(m+1)$-fat above $t_{m}$ by (11) and Lemma 3.33. Thus, using Remark 3.6 as well, we can fix a $s_{n+1} \in \operatorname{succ}_{p_{m}}\left(t_{m}\right) \backslash\left\{s_{0}, \ldots, s_{n}\right\}$ escaping the $(m+1)$-slalom $S_{m}^{k}$ such that $s_{n+1}|| r_{n}$, and $\left|s_{n+1}\right| \geq\left|r_{n}\right|$. By (4) we also have $s_{n+1} \in p_{n}$. As $s_{n+1}|| r_{n}$ and $\left|s_{n+1}\right| \geq\left|r_{n}\right|$, we obtain $p_{n}\left[s_{n+1}\right]|| r_{n}$. Hence Lemma 3.30 applied to $p_{n}\left[s_{n+1}\right], r_{n}$ and $n+1$, then Corollary 3.15] and Fact 3.13 yield a $q_{n+1} \leq p_{n}\left[s_{n+1}\right]$ and a $r_{n+1}^{\prime} \supset r_{n}$ such that $q_{n+1} \| r_{n+1}^{\prime}$ and $q_{n+1} \Vdash "\left[r_{n+1}^{\prime}\right] \subset \dot{U}_{n+1} "$, and if $t_{n+1}=\operatorname{root}\left(q_{n+1}\right)$ then

$$
\operatorname{succ}_{q_{n+1}}\left(t_{n+1}\right) \text { is } n+3 \text {-fat above } t_{n+1} \text {. }
$$

Then $r_{n+1}^{\prime}|| t_{n+1}$, hence we can clearly find an $r_{n+1}^{\prime \prime} \supset r_{n+1}^{\prime}$ such that $\left|r_{n+1}^{\prime \prime}\right| \geq$ $\left|t_{n+1}\right|$ and $r_{n+1}^{\prime \prime}|| t_{n+1}$. Finally, since $V_{n+1}$ is dense open, we can extend $r_{n+1}^{\prime \prime}$ further to obtain an $r_{n+1} \supset r_{n+1}^{\prime \prime}$ with $\left[r_{n+1}\right] \subset V_{n+1}$. Setting $p_{n+1}=\left(p_{n} \backslash\right.$ $\left.p_{n}\left[s_{n+1}\right]\right) \cup q_{n+1}$ finishes the $n+1^{\text {st }}$ step.

Now we check that the inductive assumptions are satisfied. For (11) and (2) it suffices to check that $r_{n+1}|| t_{n+1}$ and $\left|r_{n+1}\right| \geq\left|t_{n+1}\right|$, which is analogous 
to the case $n=0$ above. Items (3) and (4) follow from the structure of the fusion, as already described in Lemma 3.21. Namely, at the $n+1^{\text {st }}$ step we only modify $p_{n}$ in the 'cone' $p_{n}\left[s_{n+1}\right]$, and this cone does not contain the earlier $t_{m}$ 's, moreover, an element of $\operatorname{succ}_{p_{m}}\left(t_{m}\right)$ only 'disappears' from $p_{n}$ when it is picked as an $s_{n+1}$. Items (6), (17), and (8) are straightforward from the construction, and (5) follows from (7) and (3.3).

Let us now define $p^{\prime}=\left\{t_{m}\right\}_{m \in \omega}$. It is easy to see that $p^{\prime} \in \mathbb{P}$, since $\operatorname{succ}_{p^{\prime}}\left(t_{m}\right)$ is $m+1$-fat above $t_{m}$ for every $m$. Combining (3) and (6) we obtain

$$
p^{\prime} \leq p_{n}
$$

for every $n$, and also that $p^{\prime} \leq p$.

What remains to be shown is that $p^{\prime} \Vdash$ " $r \in \bigcap_{n} \dot{U}_{n}$ ", that is, $p^{\prime} \Vdash$ " $r \in \dot{U}_{n_{0}}$ " for every fixed $n_{0}$. Let $p^{\prime \prime} \leq p^{\prime}$ be arbitrary, then it suffices to find a $p^{\prime \prime \prime} \leq p^{\prime \prime}$ such that $p^{\prime \prime \prime} \Vdash " r \in \overline{\dot{U}}_{n_{0}}$ ". As every condition is infinite, there exists $n \geq n_{0}$ such that $t_{n} \in p^{\prime \prime}$. Then (3.4), (7), and (8) imply $p^{\prime \prime}\left[t_{n}\right] \leq p^{\prime}\left[t_{n}\right] \leq p_{n}\left[t_{n}\right]=q_{n} \Vdash "\left[r_{n}\right] \subset \dot{U}_{n} "$. Therefore, since the $U_{n}$ 's are decreasing and $r \in\left[r_{n}\right]$, we obtain $p^{\prime \prime}\left[t_{n}\right] \Vdash$ " $r \in \dot{U}_{n_{0}}$ ". Thus $p^{\prime \prime \prime}=p^{\prime \prime}\left[t_{n}\right]$ works, and this finishes the proof of the lemma.

\subsection{Putting the proof together}

Theorem 3.35 (Second Main Theorem) It is consistent with $Z F C$ that for every non-meagre set $X \subset \prod_{m \in \omega} \mathbb{Z}_{m+3}$ there is some $t \in \prod_{m \in \omega} \mathbb{Z}_{m+3}$ such that $X \cap\left(C_{E K}+t\right)$ is non-meagre in $C_{E K}+t$.

Proof. Iterate $\mathbb{P}$ of length $\omega_{2}$ with countable support over a model $V$ of the Continuum Hypothesis to obtain $V_{\alpha}$ for $\alpha \leq \omega_{2}$. If $V_{\omega_{2}} \models$ $X \subset \prod_{m \in \omega} \mathbb{Z}_{m+3}$ is non-meagre, then by an easy reflection argument there is an $\alpha<\omega_{2}$ such that $V_{\alpha} \models X \cap V_{\alpha}$ is non-meagre (see the analogous [1, Lemma 12.]). Applying Corollary 3.23 yields that in $V_{\alpha+1}$ there is some $t_{\alpha+1} \in \prod_{m \in \omega} \mathbb{Z}_{m+3}$ such that $V_{\alpha+1} \models\left(X \cap V_{\alpha}\right) \cap\left(C_{E K}+\right.$ $\left.t_{\alpha+1}\right)$ is non-meagre in $C_{E K}+t_{\alpha+1}$. Then Theorem 3.19 implies that $V_{\omega_{2}} \models$ $\left(X \cap V_{\alpha}\right) \cap\left(C_{E K}+t_{\alpha+1}\right)$ is non-meagre in $C_{E K}+t_{\alpha+1}$. Hence for the larger set we also obtain $V_{\omega_{2}} \models X \cap\left(C_{E K}+t_{\alpha+1}\right)$ is non-meagre in $C_{E K}+t_{\alpha+1}$, which completes the proof. 


\section{Open problems}

In this final section we collect the open questions.

Problem 4.1 Let $X \subset \mathbb{R}$.

$$
\forall t \in \mathbb{R} \mu_{\text {Cantor }}(X+t)=0 \Longrightarrow \lambda(X)=0 ?
$$

Problem 4.2 Is it consistent that there exist an atomless singular Borel probability measure $\mu$ such that for every $X \subset \mathbb{R}$ with $\lambda(X)>0$ there exists $t \in \mathbb{R}$ such that $\mu(X+t)>0$ ?

Problem 4.3 Is $\mathbb{P}$ of Definition 3.9 forcing equivalent to the Miller forcing?

Problem 4.4 Does Theorem 3.35 hold for $\mathbb{R}$ instead of $\prod_{m \in \omega} \mathbb{Z}_{m+3}$ ?

\section{References}

[1] T. Bartoszyński, On perfectly meager sets, Proc. Amer. Math. Soc. 130 (2002), no. 4, 1189-1195.

[2] T. Bartoszyński and H. Judah: Set theory: On the structure of the real line. A. K. Peters, Wellesley, Massachusetts, 1995.

[3] M. Burke and A. W. Miller, Models in which every nonmeager set is nonmeager in a nowhere dense Cantor set, Canad. J. Math. 57 (2005), $1139-1154$.

[4] J. P. R. Christensen, On sets of Haar measure zero in abelian Polish groups. Proceedings of the International Symposium on Partial Differential Equations and the Geometry of Normed Linear Spaces (Jerusalem, 1972). Israel J. Math. 13 (1972), 255-260 (1973).

[5] J. P. R. Christensen, Measure theoretic zero sets in infinite dimensional spaces and applications to differentiability of Lipschitz mappings. Actes du Deuxième Colloque d'Analyse Fonctionnelle de Bordeaux (Univ. Bordeaux, 1973), Publ. Dép. Math. (Lyon) 10 (1973), no. 2, 29-39.

[6] K. Ciesielski and S. Shelah, Category analogue of sup-measurability problem, J. Appl. Anal. 6 (2000), no. 2, 159-172. 
[7] U. B. Darji and T. Keleti, Covering $\mathbb{R}$ with translates of a compact set, Proc. Amer. Math. Soc. 131 (2003), no. 8, 2593-2596.

[8] R. Dougherty, J. Mycielski, The prevalence of permutations with infinite cycles, Fund. Math. 144 (1994), no. 1, 89-94.

[9] P. Erdős and S. Kakutani, On a perfect set, Colloquium Math. 4 (1957), 195-196.

[10] K. J. Falconer, The geometry of fractal sets. Cambridge Tracts in Mathematics No. 85, Cambridge University Press, 1986.

[11] B. R. Hunt, The prevalence of continuous nowhere differentiable functions, Proc. Amer. Math. Soc. 122 (1994), no. 3, 711-717.

[12] B. R. Hunt, T. Sauer and J. A. Yorke, James, Prevalence: a translationinvariant "almost every" on infinite-dimensional spaces. Bull. Amer. Math. Soc. (N.S.) 27 (1992), no. 2, 217-238.

[13] A. S. Kechris, Classical descriptive set theory. Springer-Verlag, 1995.

[14] Kunen, K.: Set theory. An introduction to independence proofs. Studies in Logic and the Foundations of Mathematics, 102. North-Holland, 1980.

[15] P. Mattila: Geometry of sets and measures in Euclidean spaces. Cambridge Studies in Advanced Mathematics No. 44, Cambridge University Press, 1995.

[16] J. C. Oxtoby: Measure and category. A survey of the analogies between topological and measure spaces. Second edition. Graduate Texts in Mathematics No. 2, Springer-Verlag, 1980.

[17] A. Rosłanowski and S. Shelah, Measured creatures, Israel J. Math. 151 (2006), 61-110.

[18] L. Zajíček, On differentiability properties of typical continuous functions and Haar null sets, Proc. Amer. Math. Soc. 134 (2006), no. 4, 1143-1151.

Rényi Alfréd Institute, Reáltanoda u. 13-15. Budapest 1053, HUNGARY

AND 
Institute of Mathematics, EÖtvös Loránd University, Pázmány PÉTer S. 1/C, Budapest 1117, Hungary

Email address: emarci@renyi.hu

www.renyi.hu/ emarci

Department of Mathematics, York University, Toronto, OnTARIO M3J 1P3, CANADA

Email address: steprans@yorku.ca 